\title{
LASER WAKEFIELD DIAGNOSTIC USING HOLOGRAPHIC LONGITUDINAL INTERFEROMETRY*
}

\author{
P. Volfbeyn, E. Esarey, and W. P. Leemans, LBNL, Berkeley, CA
}

\section{Abstract}

We propose a diagnostic technique for wakefield measurement in plasma channels. A new technique for plasma channel creation, the Ignitor Heater scheme was proposed and experimentally tested in hydrogen and nitrogen previously [1]. It makes use of two laser pulses. The Ignitor, an ultrashort (sub $100 \mathrm{fs}$ ) laser pulse, is brought to a line focus using a cylindrical lens to ionize the gas. The Heater pulse (160 ps long) is used to heat the existing spark via inverse Bremsstrahlung. The hydrodynamic shock expansion creates a partially evacuated plasma channel with a density minimum on axis. Such a channel has properties of an optical waveguide. This technique allows creation of plasma channels in low atomic number gases, such as hydrogen, which is of importance for guiding of highly intense laser pulses. Laser pulses injected into such plasma channels produce a plasma wake that has a phase velocity close to the speed of light. A discussion of plasma wake measurements, using a Longitudinal Interferometry Wakefield Diagnostic Based on Time Domain Rayleigh Refractometry [2] with Holographic Inversion, will be presented.

\section{WAKEFIELD EXCITATION IN CHANNELS}

Excitation of wakes with intense laser pulses propagating in plasma channels needs to be studied. The goal is to carry out a parametric study of wake characteristics versus plasma channel and laser pulse properties. The longitudinal and transverse plasma density profile in the channel will be measured interferometrically, as discussed previously [1]. Longitudinal femtosecond interferometry [2], [3] will be used to measure the wakefield. The technique measures the phase shift imparted by the plasma wave to an externally injected laser pulse, as a function of delay time behind the driving pulse. In this paper, the focus is on adapting the longitudinal interferometry techniques to the experiments planned at the L'OASIS laboratory and further refining them to provide an accurate and reliable measurement of the wake amplitude.

A description of the principles of the diagnostic is given in Section 3. In Section 4, it is shown that the method can be simply extended to measure a long (multiperiod) temporal record of the plasma wave evolution in a single shot. A holographic inversion technique has been developed and will be applied, that makes the interferometry technique insensitive to the effect of a phase shift common to both pulses. It therefore provides a true measure of the actual phase shift imposed on the laser beam by the plasma wake.

\footnotetext{
* Work supported by the US Department of Energy, Contract DEAC03-76SF00098.
}

\section{EXPERIMENTAL PARAMETERS}

The planned experimental parameters are: laser pulse duration $50 \mathrm{fs}, 1-10 \mathrm{~mm}$ long channel, resonant density on axis (assuming Gaussian temporal laser pulse shape) $n_{0}=7 \times 10^{17} \mathrm{~cm}^{-3}$, with a $1 \mathrm{TW}$ guided beam with guided spotsize of 10 microns. The resultant vector potential is $a_{0}=0.544$, the peak excited axial accelerating field is Ezmax $=9.35 \mathrm{GV} / \mathrm{m}$, the plasma wave density perturbation $\delta n / n=0.112$, and the peak phaseshift accumulated by a probe laser pulse co-propagating with the wake is $\Delta \phi_{\max }=0.182 \mathrm{rad} / \mathrm{mm}$ for an $800 \mathrm{~nm}$ probe pulse, and $91 \mathrm{mrad}$ for a $400 \mathrm{~nm}$ probe pulse.

\section{OPTICAL WAKEFIELD DIAGNOSTIC}

To perform phase shift measurement which are precise and insensitive to noise and fluctuations, Rayleigh's interferometric refractometer technique was applied successfully [3],[2] to measure phase shifts on the order of several mrads. This technique utilizes two laser pulses, propagating down the same path, but separated by a time delay. The leading of the two pulses traverses the plasma before the intense pulse that drives the plasma wake. The trailing pulse propagates through the plasma after the wake was produced. Thus the difference in the phaseshift acquired by the two laser pulses is due to the plasma wave. The interferometric pulses are subsequently combined in an imaging grating spectrometer. After diffracting off the grating the obtained pattern is viewed by a CCD camera at the image plane. Because of the pulse-stretching caused by the diffraction grating, the two laser pulses now overlap in time and a beat pattern is seen on the CCD. The temporal stretching $T$ can be calculated if the resolution of the spectrometer $r$ is known: $T \approx 1 / r$. For instance, $\delta \lambda=0.114 \mathrm{~nm}$ translates into pulse lengthening of $T \approx 1 /(\varnothing \delta \lambda / \lambda)=3 \mathrm{ps}$ for $800 \mathrm{~nm}$ radiation. The spatial periodicity of the beat pattern is directly related to the time separation between the two pulses.

To illustrate it with an example, assume that the two laser pulses are of the form

$$
\begin{aligned}
E(t) & =e^{\left(-(t+\Delta)^{2} / \tau^{2}\right)} \cos \left(\omega_{0}(t+\Delta)\right) \\
& +e^{\left(-t^{2} / \tau^{2}\right)} \cos \left(\omega_{0} t+\phi\right)
\end{aligned}
$$

The effect of the (perfect) grating spectrometer is equivalent to taking one half of the Fourier transform of the pulses (positive or negative frequency):

$$
\begin{array}{r}
E(\omega)=\frac{1}{2} \sqrt{\pi}\left(e^{-\left(\omega-\omega_{0}\right)^{2} \tau^{2} / 4} e^{-\left(\omega-\omega_{0}\right) \Delta}\right. \\
\left.+e^{-\left(\omega-\omega_{0}\right)^{2} \tau^{2} / 4} e^{-i \phi}\right)
\end{array}
$$


The CCD measures the power (proportional to the absolute value of the electric field squared). The CCD pattern, Fig. 1, will contain contributions from DC terms of the individual pulse amplitudes and the beating cross term with frequency domain variation as $\cos \left(\phi+\left(\omega-\omega_{0}\right) \Delta\right)$ :

$$
\begin{array}{rl}
P(\omega) \propto 2 & 2 e^{-\left(\omega-\omega_{0}\right)^{2} \tau^{2} / 2} \\
& +2 e^{-\left(\omega-\omega_{0}\right)^{2} \tau^{2} / 4} \cos \left(\phi+\left(\omega-\omega_{0}\right) \Delta\right)
\end{array}
$$

The beating contains information about the phase shift

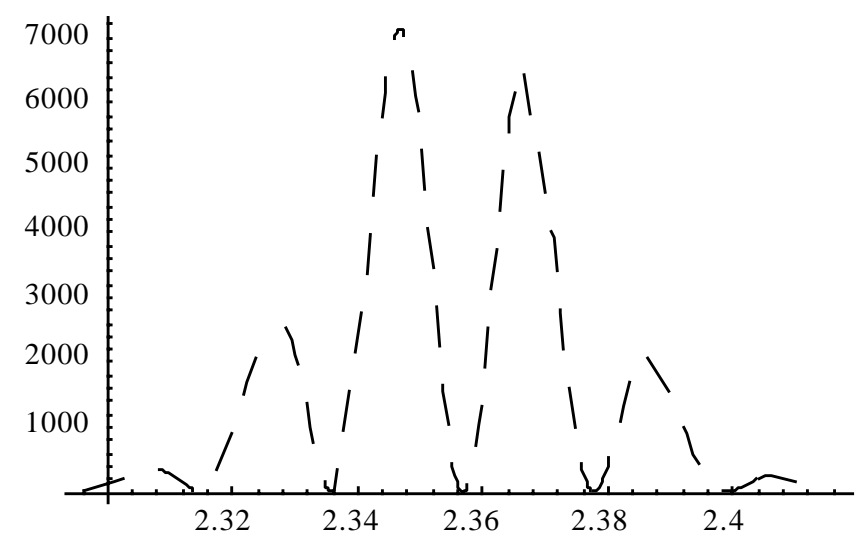

Figure 1: The spectral beat observed after the two interferometer pulses are combined on the spectrometer.

$\phi_{\max }$. Figure 2 demonstrates the difference between the beating with $\phi=0 \mathrm{mrad}$ (solid line), and $\phi=182 \mathrm{mrad}$ (dashed line).

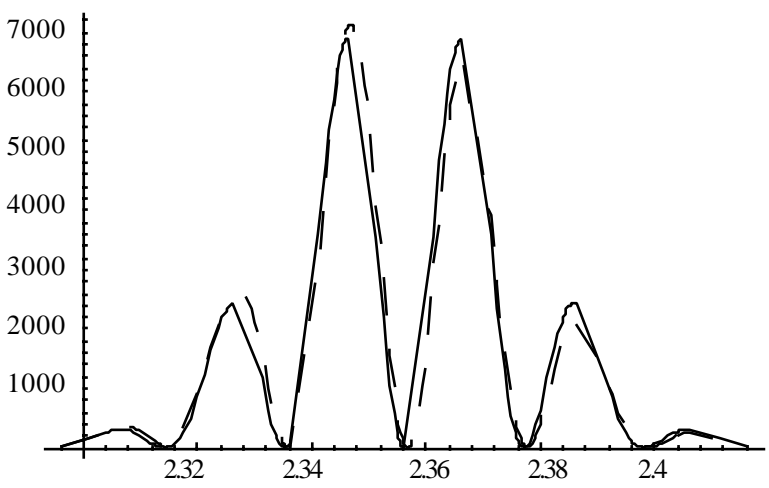

Figure 2: The interference pattern shifts in the spectral domain if the probe pulse has acquired different phaseshift than the reference pulse. $\phi=0$ mrad (solid line), and $\phi=182 \operatorname{mrad}$ (dashed line)

The phaseshift, $\phi$, can now be extracted from the beating pattern, by performing a Fourier transform of the spectrum with respect to $\left(\omega-\omega_{0}\right)$. The result of such operation is shown in Fig. 3. The three amplitude peaks correspond to the DC component and two terms at the beat frequency. The phase of the Fourier transform at the two higher frequency peaks is exactly $\pm \phi$. (a)

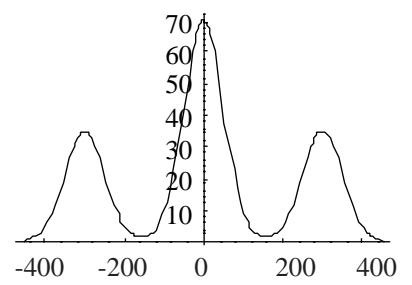

(b)

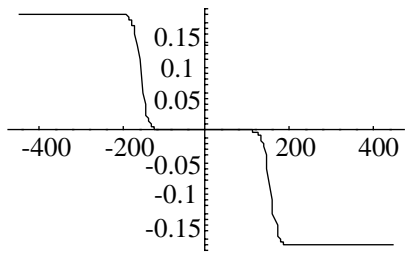

Figure 3: (a) Amplitude and (b) phase of the Fourier transform of the beat pattern of Eq. 3 with $\phi=182 \mathrm{mrad}$.

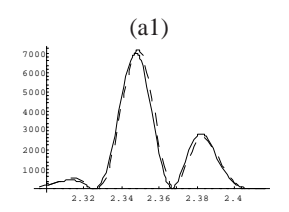

(a2)

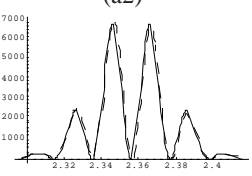

(a3)

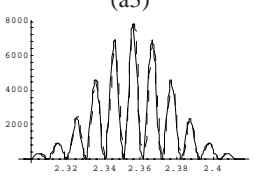

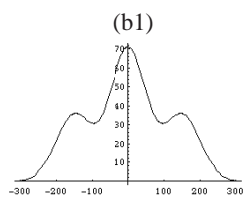

(b2)

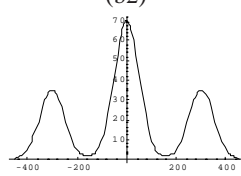

(b3)

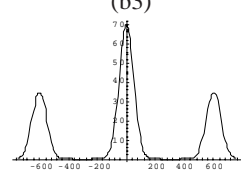

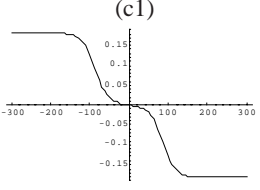

(c2)

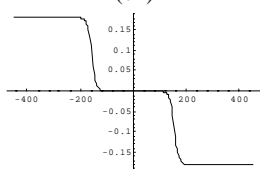

(c3)

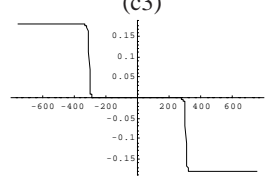

Figure 4: (a1)-(a3) Spectrometer interferometric beat pattern, (b1)-(b3) the amplitude and (c1)-(c3) phase of the Fourier transform of the beat pattern, for $\Delta=150 \mathrm{fs}, 300$ fs and $600 \mathrm{fs}$, respectively.

The time delay between the two interferometric pulses should be chosen such that the DC peak does not overlap with the two beat frequency peaks. The spectrometer beat patterns and their Fourier transforms are displayed in Figs. 4. From the figures it can be seen that when the time delay between the two pulses is too small (Fig. 4a1,b1,c1), there is considerable overlap between the peaks in the Fourier transform, and that transfers into phase error. On the other hand, if the delay is made too large, the resolution limit of the spectrometer may be reached, and the Nyquist criterion may not be satisfied. We find that the smallest value of $\Delta$ for which the peaks no longer overlap is $\Delta=5 \times t$. 


\section{TIME VARYING PHASESHIFT RETRIEVAL WITH HOLOGRAPHIC INVERSION}

Simple Fourier transformation of the spectral beat provides correct phase shift information only in the case when the phase shift is constant in time. For instance, in the case of a linearly changing (in time) phase shift, the retrieved phase has a slope that's two times smaller than the actual value. The reason for the discrepancy is that instead of taking an inverse Fourier transform of the Fourier transform of the probe pulse, the diagnostic calculates the inverse Fourier transform of the product of the probe and reference pulse transforms.

We have developed a novel theoretical algorithm for time domain Rayleigh refractometry, that relies on holographic inversion of the measured spectra. As mentioned previously, a simple Fourier transformation of the spectral beat provides correct phase shift information only in the case when the phase shift is constant in time. For instance, in the case of a linearly changing (in time) phase shift, the retrieved phase has a slope that is two times smaller than the actual [2]. The reason for the discrepancy is that instead of taking an inverse Fourier transform of the Fourier transform of the probe pulse, the diagnostic calculates the inverse Fourier transform of the product of the probe and reference pulse transforms. If the amplitude of the Fourier transform of the reference pulse is known, and if the only phase difference between the probe and reference pulses is due to the plasma wake (so that any other phase shift that is common to both pulses will cancel when a product with complex conjugate is taken), then by dividing the spectral beat by the amplitude of the reference pulse Fourier transform (square root of the spectrum of the reference pulse alone) before the Fourier transformation is calculated, the true phase shift is found. This procedure is similar to the action of the reference pulse in a hologram.

Figs. 5 presents a numerical simulation of the phaseshift retrieval with and without the holographic correction. The result of numerically calculating the inverse Fourier transform of Eq.3 with $\phi=\phi_{\max } \cos \left(\omega_{p} t+\Phi_{0}\right)$ where $\phi_{\max }=182 \mathrm{mrad}$ is expected to be close to the experimental values, is shown in Fig. 5 a). Fig. 5 b) presents the result of taking the Fourier transform after the expression of Eq. 3 was corrected for the reference pulse Fourier transform amplitude. The phaseshift retrieved without the holographic correction, Fig. 5 a), is drastically different from the actual phaseshift. The corrected retrieval, Fig. 5 b), is shown to extract the prescribed time dependent phaseshift accurately. The holographic inversion technique makes the interferometry technique insensitive to the effect of a phase shift common to both pulses, and therefore provides a true measure of the actual phase shift imposed on the laser beam by the plasma wake. We have also verified that the method can be extended to provide a single shot measurement of multiple periods of the wake pattern. Experimentally, the probe laser pulse will be made temporally longer by spec- tral filtering.

An important potential source of error is the fact that the diagnostic is unable to distinguish between Fourier limited probe pulses and pulses with a frequency chirp (quadratic phase shift). If both the probe and the reference pulses have similar frequency chirp then, after diffracting off the spectrometer grating, the probe pulse phase shift due to the wake at the front of the pulse will be mixed with that accumulated in the rear of the pulse. In the case of time varying phase shift, the retrieval will be in error. To minimize the contribution of imperfect temporal compression, the production of the blue interferometric pulses will be done in a way that ensures proper compression, provided the driving red pulse in optimally compressed.
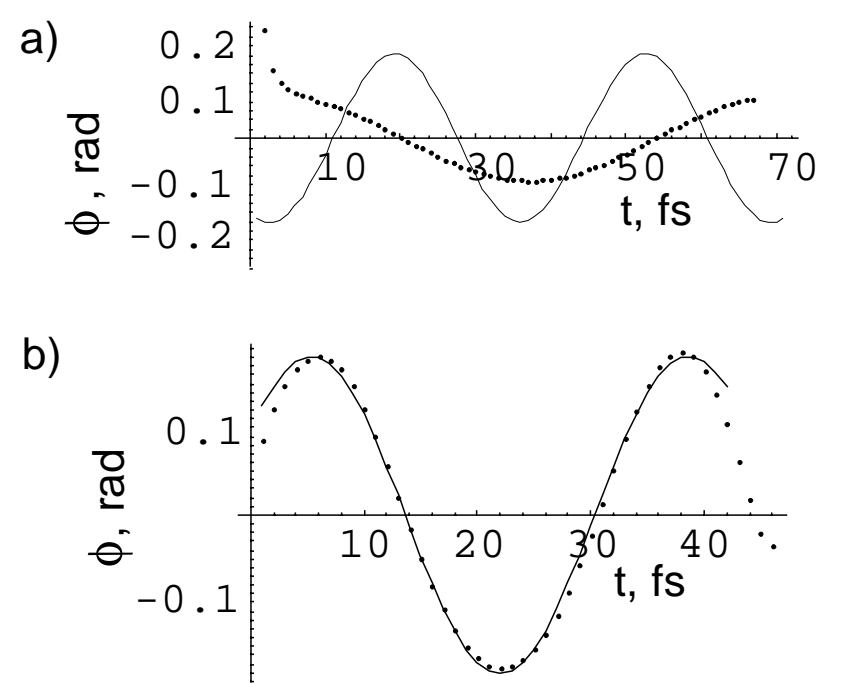

Figure 5: Retrieved phase shift (dots) a) without and b) with reference correction, compared to that prescribed (solid curves).

\section{REFERENCES}

[1] P. Volfbeyn and W. P. Leemans, "Experimental Studies on Laser Guiding with Plasma Channels," Conf. Proc. E-PAC 98, Stockholm, Sweden, p. 265, (1998)

[2] C.W. Siders et al., Phys. Rev. Lett. 76, 3570 (1996); C.W. Siders et al., IEEE Trans. Plasma Science 24, 301-305 (1996).

[3] J.R. Marquès et al., Phys. Rev. Lett. 76, 3566 (1996); J.R. Marquès et al., Phys. Rev. Lett. 78, 3463 (1997).

[4] A. Ting et al., Phys. Rev. Lett. 77, 5377 (1996).

[5] S.P. LeBlanc et al., Phys. Rev. Lett. 77, 5381 (1996).

[6] C.E. Clayton et al., Phys. Rev. Lett. 81, 100 (1998). 